\title{
Unusual course of disease and genetic profile in Li-Fraumeni syndrome-associated osteosarcoma - a case report
}

\author{
Alexander Puzik ${ }^{1 *}$ D, Markus Uhl ${ }^{2}$, Juri Ruf ${ }^{3}$, Tilmann Schumacher ${ }^{4}$ and Udo Kontny ${ }^{1,5}$
}

\begin{abstract}
Background: Osteosarcoma is a highly malignant tumour associated with numerous and complex genetic alterations like copy number alterations. Recent whole genome studies revealed distinct mutations in several candidate oncogenes.

While clinical parameters stratify osteosarcoma patients in risk groups, genetic profiles have not yet been used to tailor tumour treatment. However, specific copy number alterations seem to have a prognostic impact in osteosarcoma treatment.

Somatic TP53 gene mutation frequently occurs in sporadic osteosarcoma. When arising germline, TP53 mutation leads to Li-Fraumeni syndrome and may result in early life osteosarcoma. The effect of Li-Fraumeni syndrome on the genetic profile of osteosarcoma and the consideration of the syndrome during cancer treatment are topics of current research.

Case presentation: We report a 25 -year-old female with pelvic osteosarcoma refusing continuation of therapy. She interrupted neo-adjuvant chemotherapy according to EURAMOS-1/COSS recommendations and declined local or further adjuvant therapy. Surprisingly, she remained in sustained remission for the osteosarcoma but eventually died from newly diagnosed breast cancer. After establishment of breast cancer, we detected TP53 germline mutation and investigated the osteosarcoma material with array-CGH.
\end{abstract}

Conclusion: Genetic examination of the tumour evidenced several copy number alterations with striking differences to previously reported data. We discuss possible influences of the genetic profile on the unusual clinical course and the significance of Li-Fraumeni syndrome for the genetic profile. Specific loss of (proto-) oncogenes might have contributed to the unusual case. Further large-scale genetics of Li-Fraumeni patients combined with detailed clinical data will help to identify specific genetic risk profiles and improve treatment.

Keywords: Osteosarcoma, Li-Fraumeni syndrome, Genetics, Copy number alterations

\section{Background}

Osteosarcoma (OS) is a high-grade malignant boneproducing sarcoma and represents the most common malignant skeletal tumour of young adults with an incidence of 4/1.000.000. Prognosis is generally favourable,

\footnotetext{
* Correspondence: alexander.puzik@uniklinik-freiburg.de

'Department of Pediatric Hematology and Oncology, Medical Center -

University of Freiburg, Faculty of Medicine, University of Freiburg, Mathildenstrasse 1, D-79106 Freiburg, Germany

Full list of author information is available at the end of the article
}

but depends on several risk factors. Tumour localisation and local therapy, presence of metastasis and chemotherapy response are the most relevant prognostic factors [1]. Without local therapy the survival rates decline to approx. 10 to $20 \%$ [2]. Several research groups reported the impact of distinct sets of copy number alterations (CNA) and other genetic changes on prognosis. Smida et al. (2010) were able to predict treatment response better by genetic changes like CNA compared to the Salzer Kuntschik grading. Yen et al. (2009) identified

\section{$\triangle B M C$}

(c) The Author(s). 2021 Open Access This article is licensed under a Creative Commons Attribution 4.0 International License, which permits use, sharing, adaptation, distribution and reproduction in any medium or format, as long as you give appropriate credit to the original author(s) and the source, provide a link to the Creative Commons licence, and indicate if changes were made. The images or other third party material in this article are included in the article's Creative Commons licence, unless indicated otherwise in a credit line to the material. If material is not included in the article's Creative Commons licence and your intended use is not permitted by statutory regulation or exceeds the permitted use, you will need to obtain permission directly from the copyright holder. To view a copy of this licence, visit http://creativecommons.org/licenses/by/4.0/ The Creative Commons Public Domain Dedication waiver (http://creativecommons.org/publicdomain/zero/1.0/) applies to the data made available in this article, unless otherwise stated in a credit line to the data. 
CNA specific for recurrent or metastatic OS $[3,4]$. However, OS study groups worldwide have not introduced stratification into genetic risk groups into standard care.

Upon genetic analysis complex karyotypes with multiple numerical and structural chromosomal alterations originating from a process called chromothripsis are very frequent in OS compared to other cancers $[1,5]$. OS is one of the tumours with the highest rate of CNA and mutations [6]. Genomic instability even results in genetically diverse cell populations within a single OS. Specific CNA like deletions of pro-apoptotic genes seem to induce selection advantages and work as biological filters for other "transient" CNA [5].

Somatic mutations found in OS and other cancers very frequently affect TP53, the prototype of tumour suppressor genes. Young female adults are frequently diagnosed with TP53-mutated OS and TP53 mutation frequently occurs in very young sarcoma patients [7]. Moreover, germline TP53-mutation leading to $\mathrm{Li}$ Fraumenisyndrome (LFS) is one of the major predispositions for sarcoma development $[7,8]$. Whole genome studies of OS have identified several other somatic mutations in candidate oncogenes [9].

LFS is an autosomal-dominant inherited tumour predisposition syndrome leading to multiple, specific cancers and a determinately increased lifetime risk for cancer reflected in the current LFS or Chompret criteria $[8,10]$. Early-onset breast cancer (age 30-40) is the leading LFS cancer. Germline TP53 mutation is thought to be responsible for the initiation of oncogenesis due to the gene's numerous functions in response to stress, cell cycle control, apoptosis and DNA damage repair $[8,10]$.

Interestingly, LFS patients demonstrated shorter telomeres and increased and complex CNA reflecting germline genomic instability $[8,11]$. Array- $\mathrm{CGH}$ analyses of TP53-negative LFS families and controls have not detected any changes in CNA [11].

There is no data demonstrating specific or redundant sets of CNA in LFS patients associated with a certain profile of tumours. Regarding OS, TP53-mutated OS usually seems to harbour less mutations than TP53-wild type OS [6]. However, further data evaluating the differences between genetic profile of sporadic OS and LFSassociated OS is missing.

\section{Case presentation}

A 25-year-old female professional dancer presented to the sports medicine department due to increasing pain in the left iliac crest with radiation to the left leg. She initially noticed the pain after chiropractic treatment 6 months before (see timeline in Additional file 1). Further medical history of the patient was unremarkable. After the clinical diagnosis of lumbar syndrome was established, the sports physician prescribed acupuncture and physiotherapy. Both did not ease the pain. Next, a swelling of the left hip region appeared. Ultrasound showed no signs of arthritis but raised suspicion of a soft tissue mass. The patient was then referred to the adult oncology department.

Magnetic resonance imaging (MRI) of the pelvis revealed a large tumour of the left iliac bone with infiltration of periosteal muscles and soft tissue. Left-sided pubic bone, sacrum and sacroiliac joint were infiltrated (Fig. 1a). Skeletal scintigraphy revealed no further osseous lesions (Fig. 1b), computed tomography (CT) of chest and abdomen excluded metastases. A biopsy was performed, which resulted in diagnosis of OS, grade III of chondroblastic subtype. Despite recommendation for chemotherapy, the patient decided to perform a fasting cure and lost $6 \mathrm{~kg}$ weight.

A further MRI was done a couple of weeks later due to increasing, intolerable pain, despite treatment with opioids, cox-2-inhibitors, non-steroidal anti-inflammatory drugs and coanalgesics. Tumour size remained stable. The patient then decided to start chemotherapy according to recommendations of EURAMOS-1/COSS protocol in the paediatric oncology department.

Under chemotherapy the pain subsided within 2 months and the tumour size decreased without relevant side effects except for nausea. Due to her profession as a dancer, the patient refused to undergo local therapy by surgery or radiation and discontinued chemotherapy after 4 months. We discussed the very poor prognosis of OS without local therapy in detail with the patient.

Much to our surprise, we found no signs of tumour relapse or metastases within the next year. Imaging studies displayed a stable residual tumour mass of the left iliac bone with further decline of contrast enhancement and soft tissue portions (Fig. 1c).

Unfortunately, 16 months after the end of chemotherapy the patient first recognized a lump in her left breast. Clinical examination revealed enlarged lymph nodes ( 2 $\mathrm{cm}$ ) of the left axilla. The patient refused mammography; ultrasound indicated possible breast cancer. A couple of weeks later, ultrasound-guided biopsy of the breast lump was performed resulting in the diagnosis of invasive ductal carcinoma (G2, B5b, ER/PR 0\%, HER2/ neu score 3, MIB-1 30\%). Again, the patient declined biopsy of the lymph nodes. MRI of the chest revealed destructive carcinoma of the complete left mamma without signs of pulmonary metastases (TNM: cT2, cN1, cM0). Skeletal scintigraphy neither displayed activity of the pelvic OS nor of other bone lesions. Head MRI ruled out an initially suspected parietal metastasis with slightly increased tracer uptake (Fig. 1d). Ultrasound of the abdomen displayed a hypo-echogenic nodule of the liver.

Once again the patient refused any further treatment. Two months later, she was admitted to the gynaecologic 


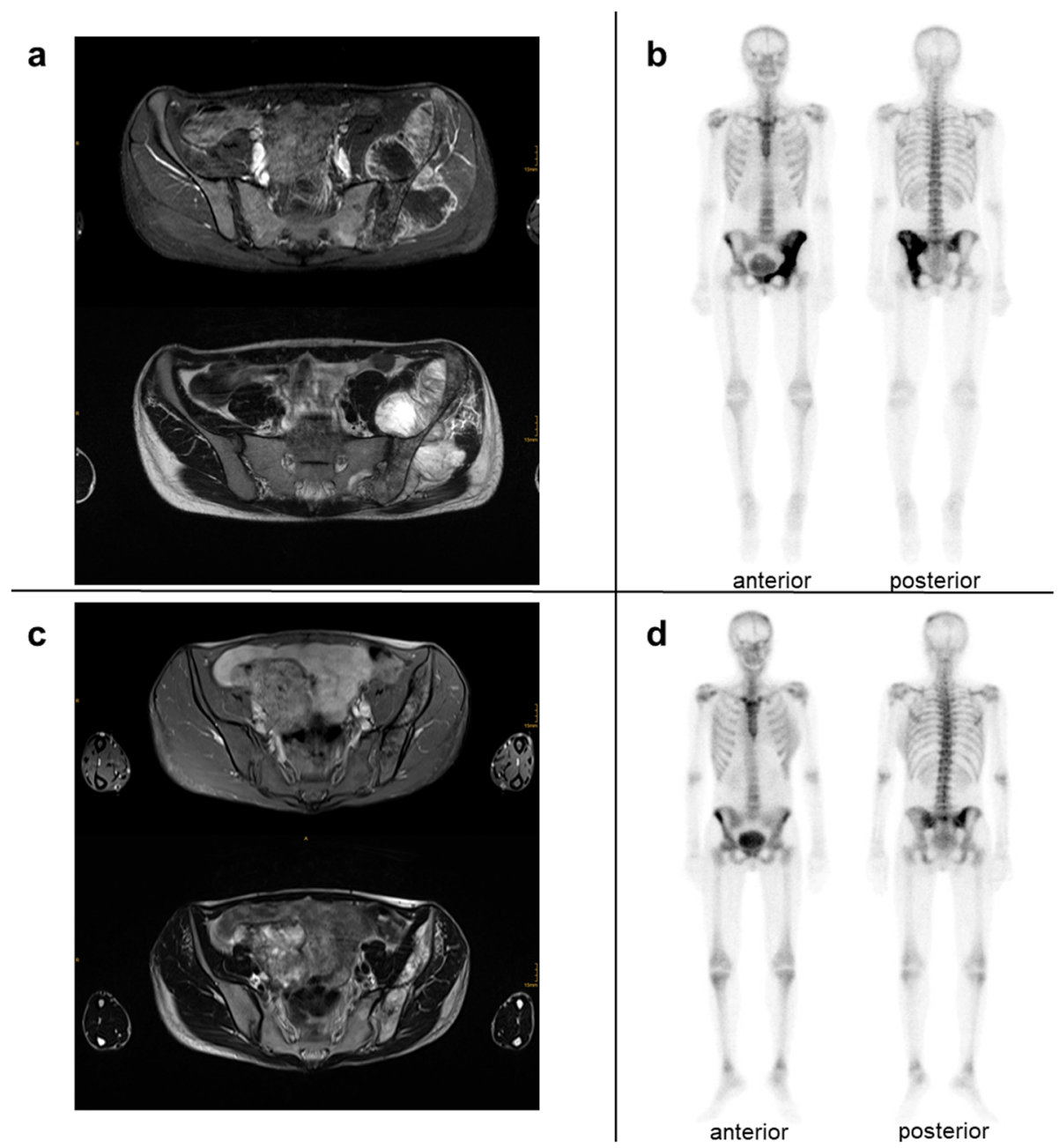

Fig. 1 Imaging results of patient at diagnosis (a/b) and last follow-up 26 months later (c/d). a Axial MRls of the pelvis showing chrondroblastic osteosarcoma of the left Os ileum with infiltration of gluteal muscles, left iliac and obturatorius internus muscle, periosteous soft tissue, Os sacrum and Os pubis with strong contrast enrichment. T ${ }_{1}$-weighted transversal MRI with i.v.-contrast-medium (gadoteriol, Prohance ${ }^{\oplus}$, Bracco) and fat saturation (above) and $\mathrm{T}_{2}$-weighted transversal MRI (bottom). $\mathbf{b}$ Aside from intensive bone turn over at the left hemipelvis, no other suspicious bone lesions are observed in initial bone scan. c Axial MRIs of the pelvis showing residual proportions of chrondroblastic osteosarcoma of the left Os ileum with decreasing contrast enrichment and signs of sclerosis and ossification of small soft tissue component. $\mathrm{T}_{1}$-weighted MRI with contrast agent and fat saturation (above) and $\mathrm{T}_{2}$-weighted MRI (bottom). $\mathbf{d}$ Bone scan demonstrating a normalisation of bone turn over at the left hemipelvis. MRI could rule out an initially suspected left parietal metastasis. Aside from an accentuation of the right sacroiliac joint due to incorrect weight bearing, no other suspicious bone lesions were observed

department with severe headaches and intermittent visual field loss. The gynaecologists interpreted findings as migraine headaches due to spontaneous improvement of pain and unremarkable MRI of the head. A couple of days later, she presented to the emergency department with sudden deterioration of her general condition, dyspnoea and decreased oxygen saturation. A CT of chest and abdomen revealed diffuse pulmonary consolidations, pericardial effusion and several hypodense hepatic nodules consistent with disseminated end-stage breast cancer. Upon further deterioration, the patient and her family agreed to withhold invasive procedures and the patient soon died of respiratory failure.
After establishing the diagnosis of breast cancer, we performed genetics for Li-Fraumeni syndrome and confirmed the diagnosis (common TP53 mutation: DNA binding domain, c.733G > A, p.Gly254Ser, heterozygous). Family history of the patient was unremarkable regarding classical Li-Fraumeni criteria, the patient's father died from a pulmonary tumour at old age.

To elucidate the very uncommon course of the OS without signs of vital tumour, despite absence of local therapy and incomplete chemotherapy, we performed array-comparative genomic hybridization $(\mathrm{aCGH})$ of the tumour material according to routine protocols (Fig. 2). 


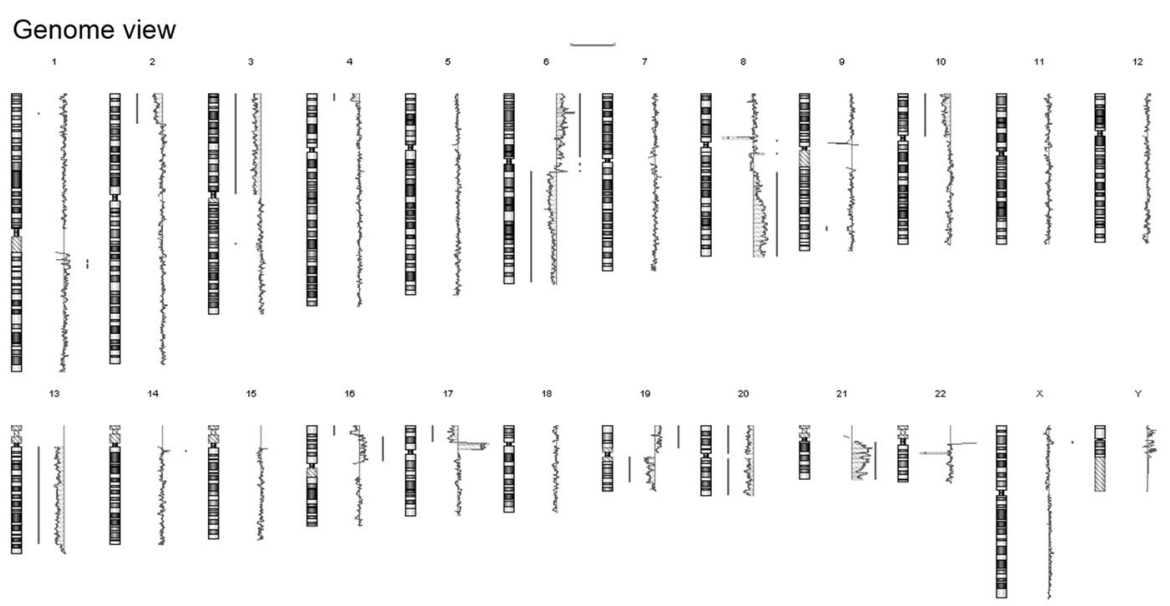

Genome view (aberrations, ADM2 algorithm)
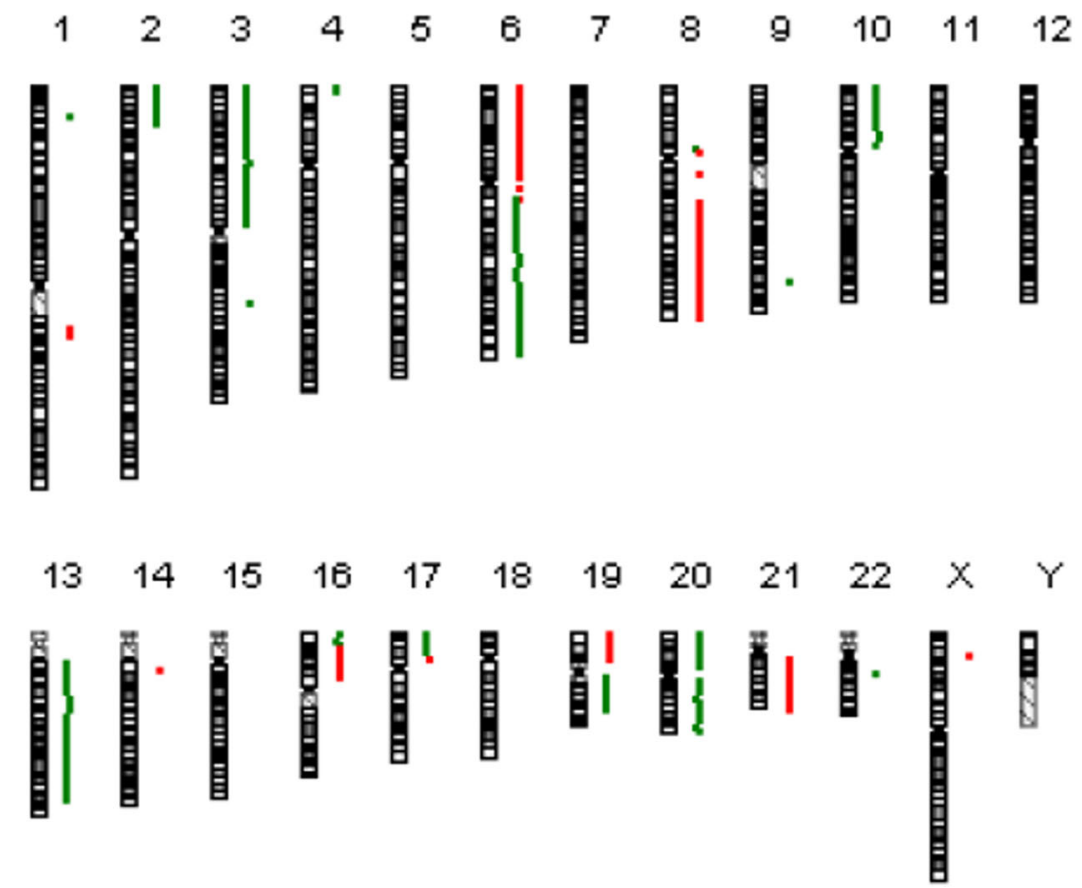

Fig. 2 Results of array-CGH. Genome view and view of aberrations (ADM2 algorithm). Copy number losses are depicted in green colour, copy number gains in red colour

Table 1 compares common CNA in OS with the individual CNA of our LFS patient.

\section{Discussion and conclusions}

OS patients without adequate local therapy display dismal survival rates with high chance of relapse and metastasis [2]. Local therapy next to chemotherapy response (and absence of metastasis) is one of the main predictors of the survival of OS patients [1, 12]. Persistent remission of OS as confirmed by lack of contrast enrichment and unremarkable diffusion parameters in MRI
(Fig. 1c) after incomplete chemotherapy without local therapy is very unusual. Moreover, the patient's TP53 mutation is usually known to be associated with an unfavourable course of OS $[7,10]$.

We elucidate below the differences in genetic profile between available data and our LFS-associated OS to raise the question as to whether a distinct genetic profile provides explanations for the unusual clinical course.

In general, we detected numerous CNA comparable to pooled data of Overholtzer et al. (2013), Rosenberg et al. (2013), Sadikovic et al. (2003) and Man et al. (2004) [1, 
Table 1 Comparison of frequently reported CNA of osteosarcoma with individual CNA of LFS patient

\begin{tabular}{|c|c|c|c|c|}
\hline \multirow[t]{3}{*}{ Chromosome } & \multicolumn{2}{|c|}{ CNA of current OS } & \multicolumn{2}{|l|}{ Frequent CNA in OS } \\
\hline & \multicolumn{4}{|c|}{ Chromosomal regions } \\
\hline & gain & loss & gain & loss \\
\hline 1 & 1923 & $1 p 36$ & $1 \mathrm{p} 32-36,1 q 21-31$ & $1 \mathrm{q} 25.1$ \\
\hline 2 & & $2 \mathrm{p} 23.2-25.2$ & & $2 q$ \\
\hline 3 & & $3 p, 3 q 22.3$ & $3 q 26$ & $3 p 14.1,3 q 13$ \\
\hline 4 & & $4 p 16.2-3$ & & $4 p 15.1$ \\
\hline 5 & & & 5p13-cen & \\
\hline 6 & 6p-cen & $6 c e n-q$ & $6 p 12-21$ & $6 p, 6 q 12,6 q 16,6 q 21-22$ \\
\hline \multicolumn{5}{|l|}{7} \\
\hline 8 & $8 q 21-24.3$ & $8 p 11.22$ & 8p, 8cen-q13, 8q12-24 & $8 p 21$ \\
\hline 9 & & $9 q 33.1$ & & $9 p 13$ \\
\hline 10 & & $10 p$ & & $10 p, \underline{10 q 22-26}$ \\
\hline 11 & & & $11 q 14$ & \\
\hline 12 & & & $12 \mathrm{p} 13-14,12 \mathrm{q} 11-14$ & \\
\hline 13 & & $13 q$ & & $13 q 12.2,13 q 14,13 q 21-31$ \\
\hline 14 & $14 q 11.2$ & & $14 q 24$ & \\
\hline \multicolumn{5}{|l|}{15} \\
\hline 16 & $16 p 12.1-13.2$ & $16 p 13.2-3$ & $16 p 13$ & \\
\hline 17 & $17 p 11.2$ & $17 p 12-13$ & $17 p 11-17 p 13,17 q 21-25$ & $17 p 13,17 q 12$ \\
\hline 18 & & & & $\underline{18 q 21-23}$ \\
\hline 19 & $19 p$ & $19 q$ & $19 p 13, \mathbf{1 9 q} \mathbf{1 2}-\mathbf{1 3}, \mathbf{1 9} \mathbf{q}$ & \\
\hline 20 & & $20 p, \mathbf{2 0 q}$ & $20 q$ & \\
\hline 21 & $21 q$ & & & \\
\hline 22 & & $22 q 11.2$ & & \\
\hline$X, Y$ & Xp22.11 & & Xp11.2-21, Xq12 & \\
\hline
\end{tabular}

Frequently reported CNA refer to data of Rosenberg et al., Sadikovic et al., Overholtzer et al. and Man et al. Similar CNA were depicted in italics, clearly opposing CNA in fat letters. Most frequently reported CNA were underlined.

5, 13, 14]. Particularly, the tumour evidenced much less copy number gains than previously reported in sporadic OS. Further, we were able to detect 25 independent chromosomal aberrations (especially losses) compared to the reported average count of independent chromosomal lesions around ten. Chromosomes 5, 7, 11, 12, 15 and 18 displayed no changes, chromosomes 1, 4, 9, 14, 22 and $\mathrm{X}$ had just minor CNA. Data regarding CNA in LFSassociated OS is not available.

Compared to the eleven most frequent aberrations out of 65 OS reported by Overholtzer et al. (2003) the current tumour evidenced five of these $(+8 \mathrm{q} 24,+6 \mathrm{p} 12,+17 \mathrm{p} 11.2$, $-6 \mathrm{q} 16,-10 \mathrm{p} 12 \mathrm{pter})$ [13]. Expanded to 28 frequent aberrations including data of Sadikovic et al. (2013) and Rosenberg et al. (2013) the current tumour demonstrated five out of 16 frequent copy number increases and five out of 12 frequent copy number losses $[1,5]$. Compared to the CNA reported by Smida et al. (2010), which were predictors of poor treatment response in OS, the current tumour evidenced only one of these (+8q24), whereas the others occurred opposing (e.g. gain of $6 \mathrm{q} 21$ instead of reported loss) or were not present $(+12 \mathrm{q} 14)$. Furthermore, these authors found poor treatment response, when more than six chromosomes were affected, especially chromosomes $2,3,5,6$, 10 and 13 [3]. This data opposes the favourable course of our highly altered OS with 16 chromosomes affected by CNA. Compared to the CNA associated with recurrence and metastasis reported by Yen et al. (2009) the current tumour evidenced three of these (-6q14.1, $-6 \mathrm{q} 16.2-22.31$, $+8 \mathrm{q} 22.3-24.3)$, whereas the others were not detectable $(-8 \mathrm{p} 23.2-12,+8 \mathrm{q} 21.12)$ or opposing $(+17 \mathrm{p} 12)$ [4]. We present a detailed comparison of frequently reported CNA in sporadic OS and CNA of our case in Table 1.

As a result of this strikingly different distribution of CNA compared to the available data, we wondered about subsequent effects on candidate oncogenes. Sadikovic et al. (2009) postulated the association between certain CNA and oncogenesis in OS discussing 
oncogenic effects of the amplification of the RUNX2 gene at 6p12.3-21.1 among others [15]. Those genes known to play a role in oncogenesis and possibly affected by CNA in our case are described in the Atlas of Genetics and Cytogenetics in Oncology and Hematology and overviewed in Additional file 2 [16].

In the context of the highly complex genetic structure of OS the following genetic alterations received further attention.

We found loss of $1 \mathrm{p} 36$ instead of the high-frequent gain of $1 \mathrm{p} 32-36$ in previous reports $[1,5,13]$. The chromosomal region harbours several genes associated with migration, proliferation and adhesion, e.g. $Y B X-1$ and ARID1A. YBX-1 (involved in splicing, transcriptional regulation, translation, chromatin remodelling and DNA repair) was usually overexpressed in OS and associated with a higher proliferative index [17]. ARID1A is a chromatine modifier and known oncogene in OS [9].

Loss of 20q and 19q has rarely been reported in OS in contrast to high-frequent gains of these regions $[1,5,13]$. Both regions comprise several candidate proto-oncogenes (20q: NKX2.2, PCNA, PLCB1, DNMT3B, E2F1, SRC, TPX2, CHD6, PLCG1, EYA2, MYBL2, UBE2L, CSE1L, SNAI1, AVRKA, TNFRSFCB, PTK6; 19q: FXYD3, FXYD5, ACTN4, AKT2, AXL, CEACAM1, CEACAM5, CIC, CXCL17, PAF1, TGFB1, CADM4, LYPD3, PLAUR, BBC3, MARK4, RELB, SLC1A5, AKT1S1, ATF5, BAX, BCL2L12, CARD8, EMP, MIR150, RUVBL2) as well as some oncogenes already known to have tumour promoting effects in OS (20q: BLCAP, GNAS; 19q: AKT2) [18, 19].

Next, we discuss the impact of LFS on genetic profile of OS and outcome. To the best of our knowledge, studies comparing genetic profile and outcome between LFS-associated OS and sporadic OS are not available. LFS itself is associated with genomic instability determined by TP53 mutation. The mutation of TP53 has certainly laid the foundation for development of random genomic alterations in subsequent tumours and is an important, but not mandatory part of OS development $[5,7,8,11]$. Clearly conflicting with our data, Bousquet et al. (2016) reported fewer mutations in TP53-mutated OS (without germline TP53-mutation) than in wild type OS [6]. Therefore, one might ask whether different time points and origins of TP53 mutation (germline vs. somatic) have an impact on the evolution of genetic profile of OS and other typical LFS tumours. Finally, even if all of the above-mentioned alterations have arisen by chance, such a genetic profile might simply be specific for LFS-associated OS. The influence of genetic profiles of OS on prognosis has already been reported $[3,4,15]$.

In conclusion, we report on a very unusual course of OS in a patient with Li-Fraumeni syndrome remaining in persistent remission after incomplete chemotherapy and without local therapy. Furthermore, we tried to specify differences between CNA in reported OS cases and our case and asked about a potential role of LFS for our findings. In our case, certain losses of (proto-) oncogenes might have played a major role in disease progression. Variations in therapy response, sensitivity to therapy and outcome are well known during treatment of cancers of other tumour predisposition syndromes like trisomy 21 or ataxia teleangiectasia.

International analysis of combined genetics and clinical data of LFS studies like the Clinical and Genetic Studies of Li-Fraumeni Syndrome of the US National Cancer Institute (NCT01443468) or the Li-FraumeniSyndrome-Cancer-Predisposition-Syndrome Registry 01 (LFS-CPS-R01) of the German Society of Pediatric Hematology and Oncology (GPOH) [20] will result in the identification of a specific genetic profile of LFS tumours. These genetic profiles will improve stratification of therapy and the outcome.

\section{Abbreviations}

aCGH: Array-comparative genomic hybridization; CNA: Copy number alterations; CT: Computed tomography; LFS: Li-Fraumeni syndrome; MRI: Magnetic resonance imaging; OS: Osteosarcoma

\section{Supplementary Information}

The online version contains supplementary material available at https://doi. org/10.1186/s13053-021-00202-0.

\section{Additional file 1. Patient's timeline}

Additional file 2. Detailed list of potentially relevant genes affected by different CNA profile. List of potentially affected genes due to CNA and potentially affected genes due to differences to previously reported CNA. Validated (candidate) oncogenes in OS according to Rickel et al. (2017) were depicted in fat letters.

\section{Acknowledgements}

We thank Daniel Baumhoer, bone tumour reference center, Basel, Switzerland, for performing aCGH analysis and supporting us with analysis.

\section{Authors' contributions}

AP conceived of, drafted and finalized the manuscript. He interpreted genetic examinations with the support of Daniel Baumhoer and was attending physician. MU carried out and interpreted radiologic studies. JR and TS carried out and interpreted bone scans. UK helped to draft, reviewed the manuscript and was attending consultant. All authors read and approved the final manuscript.

\section{Funding}

The authors received no specific funding from any funding agency in the public, commercial, or not-for-profit sectors. Open Access funding enabled and organized by Projekt DEAL.

\section{Availability of data and materials}

Data sharing is not applicable to this article as no datasets were generated or analysed during the current study.

\section{Declarations}

Ethics approval and consent to participate Not applicable. 


\section{Consent for publication}

Written informed consent was obtained from the patient for publication of this Case report and any accompanying images. A copy of the written consent is available for review by the Editor of this journal.

\section{Competing interests}

The authors declare that they have no competing interests.

\section{Author details}

'Department of Pediatric Hematology and Oncology, Medical Center University of Freiburg, Faculty of Medicine, University of Freiburg, Mathildenstrasse 1, D-79106 Freiburg, Germany. ${ }^{2}$ Division of Pediatric Radiology, Department of Radiology, Medical Center - University of Freiburg, Faculty of Medicine, University of Freiburg, Mathildenstrasse 1, D-79106 Freiburg, Germany. ${ }^{3}$ Department of Nuclear Medicine, Medical Center University of Freiburg, Faculty of Medicine, University of Freiburg, Hugstetter Strasse 55, D-79106 Freiburg, Germany. ${ }^{4}$ Practice for Nuclear Medicine, Wirthstr. 11, D-79110 Freiburg, Germany. ${ }^{5}$ Department of Pediatric Hematology, Oncology and Stem Cell Transplantation, RWTH Aachen, Pauwelsstrasse 30, D-52074 Aachen, Germany.

Received: 2 August 2021 Accepted: 10 October 2021

Published online: 20 October 2021

\section{References}

1. Rosenberg AE, Cleton-Jansen AM, de Pineux G, Deyrup AT, Hauben E, Squire J. Conventional Osteosarcoma. In: Fletcher CDM, Bridge JA, Hogendoorn PC, editors. WHO classification of tumours of soft tissue and bone. 4th ed. Lyon: IARC; 2013. p. 282-8.

2. Jaffé N, Carrasco H, Raymond K, Ayala A, Eftekhari F. Can cure in patients with osteosarcoma be achieved exclusively with chemotherapy and abrogation of surgery? Cancer. 2002;95(10):2202-10. https://doi.org/10.1002/ cncr.10944.

3. Smida J, Baumhoer D, Rosemann M, Walch A, Bielack S, Poremba C, et al. Genomic alterations and allelic imbalances are strong prognostic predictors in osteosarcoma. Clin Cancer Res. 2010;16(16):4256-67. https://doi.org/10.11 58/1078-0432.CCR-10-0284

4. Yen CC, Chen WM, Chen TH, Chen WYK, Chen PCH, Chiou HJ, et al. Identification of chromosomal aberrations associated with disease progression and a novel 3q13.31 deletion involving LSAMP gene in osteosarcoma. Int J Oncol. 2009;35(4):775-88. https://doi.org/10.3892/ijo_ 00000390.

5. Sadikovic B, Park PC, Selvarajah S, Zielenska M. Array comparative genomic hybridization in osteosarcoma. In: Banerjee D and Shah SP, editors. Array comparative genomic hybridization: protocols and applications. Methods Mol Biol 2013;973: 227-246.

6. Bousquet M, Noirot C, Accadbled F, Sales de gauzy J, Castex MP, Brousset P, et al. Whole-exome sequencing in osteosarcoma reveals important heterogeneity of genetic alterations. Ann Oncol. 2016;27(4):738-44. https:// doi.org/10.1093/annonc/mdw009

7. Ognjanovic S, Olivier M, Bergemann TL, Hainaut P. Sarcomas in TP53 germline mutation carriers. Cancer. 2012;118:1387-96.

8. Malkin D. Li-Fraumeni syndrome. In: Fletcher CDM, Bridge JA, Hogendoorn PC, editors. WHO classification of tumours of soft tissue and bone. 4th ed. Lyon: IARC; 2013. p. 379-81.

9. Rickel K, Fang F, Tao J. Molecular genetics of osteosarcoma. Bone. 2017;102: 69-79.

10. Sorrell AD, Espenschied CR, Culver JO, Weitzel JN. TP53 testing and liFraumeni syndrome: current status of clinical applications and future directions. Mol Diagn Ther. 2013;17(1):31-47.

11. Silva AG, Krepischi AC, Pearson PL, Hainaut P, Rosenberg C, Achatz MI. The profile and contribution of rare germline copy number variants to cancer risk in li-Fraumeni patients negative for TP53 mutations. Orphanet J Rare Dis. 2014;9:63.

12. Meazza C, Luksch R, Daolio P, Podda M, Luzzati A, Gronchi A, et al. Axial skeletal osteosarcoma: a 25-year monoinstitutional experience in children and adolescents. Med Oncol. 2014;31(4):875

13. Overholtzer M, Rao PH, Favis R, Lu XY, Elowitz MB, Barany F, et al. The presence of p53 mutations in human osteosarcomas correlates with high levels of genomic instability. PNAS. 2003;100(20):11547-52.
14. Man TK, Lu XY, Jaeweon K, Perlaky L, Harris CP, Shah S, et al. Genome-wide array comparative genomic hybridization analysis reveals distinct amplifications in osteosarcoma. BMC Cancer. 2004;4:45. https://doi.org/10.11 86/1471-2407-4-45.

15. Sadikovic B, Yoshimoto M, Chilton-MacNeill S, Thorner P, Squire JA, Zielenska M. Identification of interactive networks of gene expression associated with osteosarcoma oncogenesis by integrated molecular profiling. Hum Mol Genet. 2009;18(11):1962-75.

16. Atlas of Genetics and Cytogenetics in Oncology and Hematology. http://atla sgeneticsoncology.org. Last accessed 30 October 2020.

17. Oda Y, Sakamoto A, Shinohara N, Ohga T, Uchiumi T, Kohno K, et al. Nuclear expression of YB-1 protein correlates with P-glycoprotein expression in human osteosarcoma. Clin Cancer Res. 1998:4(9):2273-7.

18. Su HC, Zhao YH, Fan DG, Fan QY, Zhang P, Wen YH, et al. Relationship between expression of BLCAP protein and malignancy of osteosarcoma. $X_{i}$ Bao Yu Fen Zi Mian Yi Xue Za Zhi. 2003;19(5):465-6.

19. Carter JM, Inwards CY, Jin L, Evers B, Wenger DE, Oliveira AM, et al. Activating GNAS mutations in parosteal osteosarcoma. Am J Surg Pathol. 2014;38(3):402-9.

20. Li-Fraumeni-Syndrome-Cancer-Predisposition-Syndrome Registry 01 (Version 2017-12-29) https://www.kinderkrebsinfo.de/fachinformationen/studienporta I/pohkinderkrebsinfotherapiestudien/krebspraedispositionsregister/index ger.html Last accessed 30 October 2019.

\section{Publisher's Note}

Springer Nature remains neutral with regard to jurisdictional claims in published maps and institutional affiliations.

Ready to submit your research? Choose BMC and benefit from:

- fast, convenient online submission

- thorough peer review by experienced researchers in your field

- rapid publication on acceptance

- support for research data, including large and complex data types

- gold Open Access which fosters wider collaboration and increased citations

- maximum visibility for your research: over $100 \mathrm{M}$ website views per year

At $\mathrm{BMC}$, research is always in progress.

Learn more biomedcentral.com/submissions 